\title{
Scavenging of blood glutamate for enhancing brain-to-blood glutamate efflux
}

\author{
YUNHONG LI ${ }^{1 *}$, XIAOLIN HOU ${ }^{2 *}$, QI QI ${ }^{1 *}$, LE WANG $^{2 *}$, LAN LUO $^{3}$, SHAOQI YANG $^{2}$, \\ YUMEI ZHANG $^{1}$, ZHENHUA MIAO ${ }^{1}$, YANLI ZHANG ${ }^{1}$, FEI WANG ${ }^{1}$, HONGYAN WANG $^{1}$, \\ WEIDONG HUANG ${ }^{1}$, ZHENHAI WANG $^{2}$, YING SHEN ${ }^{4}$ and YIN WANG $^{1}$ \\ ${ }^{1}$ Department of Neurobiology of Basic Medical College, Center of Scientific Technology, Key Laboratory of \\ Cranial Cerebral Diseases, Ningxia Medical University; ${ }^{2}$ General Hospital of Ningxia Medical University, \\ Yinchuan, Ningxia 750004; ${ }^{3}$ Department of Cell Biology and Medical Genetics, Kunming Medical University, \\ Kunming, Yunnan 650500; ${ }^{4}$ Department of Neurobiology, Key Laboratory of Medical Neurobiology of \\ the Ministry of Health, Zhejiang Province Key Laboratory of Neurobiology, Zhejiang \\ University School of Medicine, Hangzhou, Zhejiang 310058, P.R. China
}

Received May 24, 2013; Accepted November 7, 2013

DOI: $10.3892 / \mathrm{mmr} .2013 .1793$

\begin{abstract}
The presence of excess glutamate in the brain interstitial fluid characterizes several acute pathological conditions of the brain, including traumatic brain injury and stroke. It has been demonstrated that it is possible to eliminate excess glutamate in the brain by decreasing blood glutamate levels and, accordingly, accelerating the brain-to-blood glutamate efflux. It is feasible to accomplish this process by activating blood resident enzymes in the presence of the respective glutamate cosubstrates. In the present study, several glutamate cosubstrates and cofactors were studied in an attempt to identify the optimal conditions to reduce blood glutamate levels. The administration of a mixture of $1 \mathrm{mM}$ pyruvate and oxaloacetate (Pyr/Oxa) for $1 \mathrm{~h}$ decreased blood glutamate levels by $\leq 50 \%$. The addition of lipoamide to this mixture resulted in a further reduction in blood glutamate levels of $>80 \%$. In addition, in vivo experiments showed that lipoamide together with Pyr/Oxa is able to decrease blood glutamate levels to a greater extent than Pyr/Oxa alone, and accordingly, this enhances the glutamate efflux from the brain to the blood. These results may outline a novel neuroprotective strategy with increased effectiveness for the removal of excess brain glutamate in various neurodegenerative conditions.
\end{abstract}

Correspondence to: Professor Yin Wang, Department of Neurobiology, Basic Medical College, Ningxia Medical University, 1160 Shengli Street, Yinchuan, Ningxia 750004, P.R. China

E-mail: yin-wang@hotmail.com

${ }^{*}$ Contributed equally

Key words: blood-brain-barrier, glutamate scavenging, pyruvate/oxaloacetate, lipoamide

\section{Introduction}

Glutamate, the major excitatory amino acid neurotransmitter in the central nervous system, is involved in the pathological process of excitotoxicity in which neuronal cells are damaged and killed by excess glutamate present in the brain fluid following various acute and chronic neurodegenerative disorders, including stroke, traumatic brain injury, amyotrophic lateral sclerosis, brain tumors, and human immunodeficiency virus (HIV) dementia. Accordingly, strategies to interfere with glutamate activity, including the inhibition of glutamate synthesis, blocking its release from presynaptic terminals, antagonizing its interactions with postsynaptic receptors and accelerating its reuptake from the synaptic cleft, have predominated recent research in the area of neuroprotection. However, all of these strategies have proved to be ineffective $(1,2)$.

The failures of the aforementioned strategies prompted the proposal of an alternative approach to the neutralization of the deleterious effects of glutamate, which is to cause an increased pumping of brain cerebrospinal fluid (CSF) glutamate into the blood in order to accelerate glutamate efflux from the brain into the blood across the blood-brain-barrier. Several examples are available, which show that the decrease of amino acid levels in the blood reduces their concentration in the CSF in parallel. The administration of asparaginase to the blood decreases asparagine levels in plasma and in the CSF (3). To achieve a similar objective for glutamate, it was hypothesized that it may be feasible to boost the brainto-blood efflux of glutamate by decreasing its levels in the blood.

In previous studies $(4,5)$, we have demonstrated that such a decrease occurs in vitro and in vivo, upon activation of the blood resident enzymes glutamate-pyruvate transaminase (GPT) and glutamate-oxaloacetate transaminase (GOT), which transform glutamate into $\alpha$-ketoglutarate in the presence of the respective glutamate cosubstrates, pyruvate (Pyr) and oxaloacetate (Oxa). The activation of GPT by Pyr and 
GOT by Oxa causes a decrease in the blood levels of glutamate. This decrease was observed to accelerate the efflux of glutamate from the brain to the blood by increasing the driving force for the brain-to-blood glutamate efflux, and thereby causing a decrease in glutamate levels in the CSF. This strategy was assessed in animal models of closed head injury, stroke, brain glioma and amyotrophic lateral sclerosis, in which it demonstrated highly significant neuroprotective effects (4).

As the ability of Pyr/Oxa to decrease blood glutamate is limited, with the maximum reduction being $50 \%$, the present study investigated the effects of certain GPT- and GOT-related compounds, in regard to their blood glutamate scavenging ability, in order to achieve optimal conditions for further decreasing the blood levels of glutamate.

\section{Materials and methods}

Chemicals. All chemicals were purchased from Sigma-Aldrich (Shanghai, China). The procedures of the animal experiments performed in this study were approved by the Animal Care Committee of Ningxia Medical University (Ningxia, China).

In vitro blood glutamate scavenging. Blood was collected retroorbitally from specific pathogen-free Sprague Dawley (SD) rats weighing 200-250 g, which were obtained from the Animal Center of Ningxia Medical University (Ningxia, China). The animals were incubated at $37^{\circ} \mathrm{C}$ in the presence of injected Pyr, Oxa or other chemicals at different concentrations. Blood aliquots were then taken at different time-points in order to measure the glutamate levels. In order to study the depletion of glutamate from the blood cell pool, the blood was centrifuged at $1,300 \mathrm{x}$ for $7 \mathrm{~min}$ at $4^{\circ} \mathrm{C}$ and the cell pellet was resuspended in Ringer-HEPES buffer containing $2.75 \mathrm{mM}$ glucose, $5 \mathrm{mM}$ HEPES, $0.15 \mathrm{M} \mathrm{NaCl}, 2 \mathrm{mM} \mathrm{CaCl}_{2}$, $0.2 \mathrm{mM} \mathrm{MgCl}_{2} \cdot 6 \mathrm{H}_{2} \mathrm{O}, 5 \mathrm{mM} \mathrm{KCl}$ and $6 \mathrm{mM} \mathrm{NaHCO}$ ( $\left.\mathrm{mH} 7.4\right)$. It was then washed three times by centrifugation and resuspension. Glutamate depletion was achieved by supplementing the blood cell pool, maintained at $37^{\circ} \mathrm{C}$, with $1 \mathrm{mM} \mathrm{Pyr} / \mathrm{Oxa}$ or other chemicals added every $15 \mathrm{~min}$, and the glutamate value was measured at different time-points.

Glutamate analysis. Blood samples were deproteinated by adding an equal volume of ice-cold $1 \mathrm{M}$ perchloric acid (PCA) and then centrifuging the samples at $16,000 \mathrm{x}$ g for $10 \mathrm{~min}$ at $4^{\circ} \mathrm{C}$. The pellet was discarded and the supernatant collected, adjusted to $\mathrm{pH} 7.2$ with $2 \mathrm{M} \mathrm{K}_{2} \mathrm{CO}_{3}$, and, when required, stored at $-20^{\circ} \mathrm{C}$ for later analysis. The glutamate concentration was measured in the supernatant using the fluorometric method by Graham and Aprison (6). A $20 \mu \mathrm{l}$ aliquot of the PCA supernatant was added to $480 \mu \mathrm{l}$ reaction buffer containing 15 units of glutamate dehydrogenase in $0.2 \mathrm{mM}$ nicotinamide adenine dinucleotide (NAD), 0.3 M glycine and $0.25 \mathrm{M}$ hydrazine hydrate adjusted to $\mathrm{pH} 8.6$ with $1 \mathrm{~N} \mathrm{H}_{2} \mathrm{SO}_{4}$. Following incubation for 30-45 $\mathrm{min}$ at room temperature, fluorescence was measured at $460 \mathrm{~nm}$ with excitation at $350 \mathrm{~nm}$. A glutamate standard curve was established with concentrations ranging between 0 and $6 \mu \mathrm{M}$. All determinations were performed at least in duplicate. The results are expressed as the mean \pm standard deviation.
Intravenous injections. In accordance with a previously described method (4), female SD rats (250-300 g) were anesthesized by intraperitoneal injection of urethane $(0.125 \mathrm{~g} / 0.2 \mathrm{ml}$ per $100 \mathrm{~g}$ body weight). Catheterization of the tail vein (for drug injections) and of the femoral vein (for blood aliquot withdrawals) were performed using PE10 polyethylene tubing linked to PE50 polyethylene tubing (Smiths Medical, Barking, UK). All catheters were secured with 5-0 silk thread (Smiths Medical) and flushed with heparin (3-5 $\mu \mathrm{l}$ of $182 \mathrm{U} / \mathrm{ml})$. The body temperature was maintained using a lamp and the rectal temperature was monitored. Intravenous injections of $\mathrm{Pyr} / \mathrm{Oxa}$ and lipoamide diluted in phosphate-buffered saline (PBS) were performed at a rate of $0.05 \mathrm{ml} / \mathrm{min}$ for $30 \mathrm{~min}$ with a Pharmacia pump P-1 (Pharmacia, Uppsala, Sweden). During and following the injections, aliquots of $150 \mu \mathrm{l}$ blood were removed from the femoral vein every $15 \mathrm{~min}$.

Intracerebroventricular injections. A steel cannula made from a $27 \mathrm{G}$ needle was implanted into the right lateral ventricle of the rat using the following stereotactic coordinates: $0.8 \mathrm{~mm}$ posterior to the bregma; $1.4 \mathrm{~mm}$ lateral from the midline; $4 \mathrm{~mm}$ below the skull surface and $3.5 \mathrm{~mm}$ from the dura. A total volume of $11 \mu \mathrm{l}\left[{ }^{3} \mathrm{H}\right]$ glutamate solution in PBS was injected into the lateral ventricle in $\sim 3$ min through the implanted cannula using a Hamilton syringe $(25 \mu \mathrm{l})$ connected to a PE20 tube filled with the solution. For radioactivity determination, $50-\mu 1$ blood samples, collected from the femoral vein, were diluted in $500 \mu 1 \mathrm{H}_{2} \mathrm{O}$ and added to a 16-ml scintillator (PerkinElmer Lifesciences, Waltham, MA, USA). The measured counts per minute (cpm) values were corrected for quenching as determined by comparing the measured cpm of a set volume of $\left[{ }^{3} \mathrm{H}\right]$ glutamate added to water or to diluted blood.

Statistical analysis. Results are presented as the mean \pm standard error of the mean. Data were analyzed by Student's t-test. $\mathrm{P}<0.05$ was considered to indicate a statistically significant difference.

\section{Results}

Scavenging blood glutamate with Pyr/Oxa in vitro. The optimal concentration and time-point for minimizing blood glutamate levels with Pyr/Oxa were evaluated. Fig. 1 illustrates the changes in blood glutamate levels upon addition of various concentrations of the mixture of Pyr/Oxa, at $\mathrm{t}=0,20,30,40$, and $60 \mathrm{~min}$. The results show that the blood glutamate levels were reduced by $\leq 50 \%$ by basal levels of the blood resident transaminases GPT and GOT with addition of a mixture of $1 \mathrm{mM}$ Pyr/Oxa for $60 \mathrm{~min}$. Pyr/Oxa act in synergy to decrease glutamate levels but their effects are limited by the build-up of $\alpha$-ketoglutarate, which facilitates the back reaction of glutamate formation.

Impact of cofactors on glutamate depletion. Blood contains resident enzymes, such as GPT (substrate, Pyr), GOT (substrate, Oxa) and glutamate dehydrogenase (substrate, GDH). In the presence of their substrates, glutamate is transformed into $\alpha$-ketoglutarate by these transaminases (Fig. 2). The products and cofactors of these reactions may affect the efficiency of the glutamate conversion. 
A

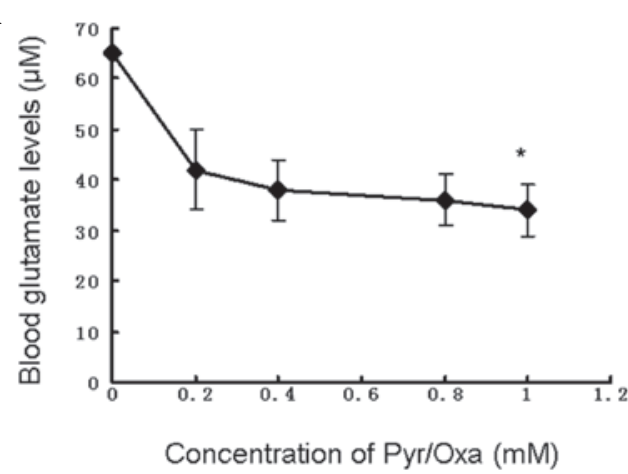

B

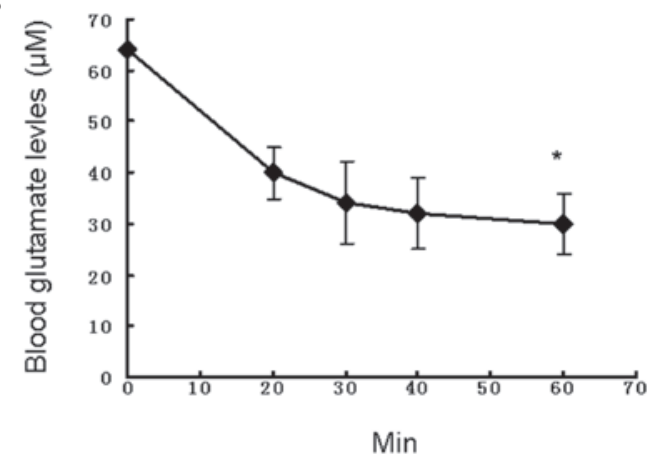

Figure 1. In vitro scavenging of blood glutamate upon supplementation with various concentrations of pyruvate and oxaloacetate (Pyr/Oxa) over (A) 60 min or (B) at different time-points. Each point represents the average of eight experiments \pm standard error of the mean. ${ }^{*} \mathrm{P}<0.05$ compared with basal glutamate level.

In the presence of Pyr, blood glutamate is converted to $\alpha$-ketoglutarate by GPT, and Pyr to alanine (Fig. 2). However, this process is reversible as the enzyme operates equally well in both directions. As shown in Fig. 3, the addition of alanine diminished the ability of Pyr to decrease the glutamate concentration. As the concentration of alanine was increased, the glutamate conversion into $\alpha$-ketoglutarate was reduced.

In regard to the fact that glutamate is converted into $\alpha$-ketoglutarate and that the latter is able to drive the GPT- and GOT-catalyzed back reactions, thereby limiting the conversion of glutamate, the present study investigated whether $\alpha$-ketoglutarate, the common coproduct of GPT and GOT, was able to serve as a substrate of $\alpha$-ketoglutarate dehydrogenase, which converts $\alpha$-ketoglutarate into succinyl coenzyme A (CoA) in the presence of CoA and NAD (Fig. 2). Therefore, the consequences of the addition of CoA and NAD on glutamate conversion were assessed. In addition, the effects of the addition of pyridoxal phosphate, which is the cofactor of GPT/GOT, and of cocarboxylase and lipoamide, which are cofactors of $\alpha$-ketoglutarate dehydrogenase (Fig. 2), were examined. As shown in Fig. 4, the extent of glutamate scavenging in the blood increases with increasing concentration of Pyr/Oxa. Pyridoxal phosphate exerted a marginal effect, while cocarboxylase decreased the glutamate conversion. However, lipoamide increased the conversion from a value of $55 \%$ in the control (1 mM Pyr/Oxa) to $83 \%$.

Since, as observed in Fig. 4, lipoamide appeared to contribute to the scavenging of blood glutamate, the dose-efficiency correlation was investigated by applying various concentrations of lipoamide to blood. Fig. 5 shows that a maximal extent of glutamate conversion was obtained with $100 \mu \mathrm{M}$ lipoamide.

As blood contains, in addition to GPT and GOT, other enzymes, such as GDH, the possible contribution of GDH to the scavenging of glutamate was investigated. GDH is a multimeric enzyme that uses NAD or nicotinamide adenine dinucleotide phosphate (NADP) as cofactors to transform glutamate into $\alpha$-ketoglutarate and ammonia. It is allosterically activated by leucine and ADP (Fig. 2). Therefore, the glutamate scavenging effects of NAD, ADP and leucine (all at $1 \mathrm{mM}$ ) were assessed. Fig. 6 illustrates the effects of the repeated addition of $1 \mathrm{mM}$ NAD to blood on glutamate levels in the cell and plasma compartments: While a decrease in glutamate levels was found to occur in the blood cells, an increase in glutamate

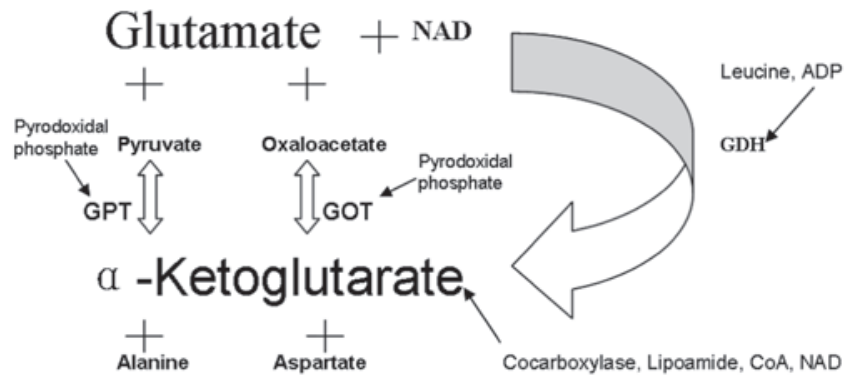

Figure 2. Schematic of the chemical reactions leading to glutamate depletion. GPT, glutamate-pyruvate transaminase; GOT, glutamate-oxaloacetate transaminase; NAD, nicotinamide adenine dinucleotide; GDH, glutamate dehydrogenase; CoA, coenzyme A.

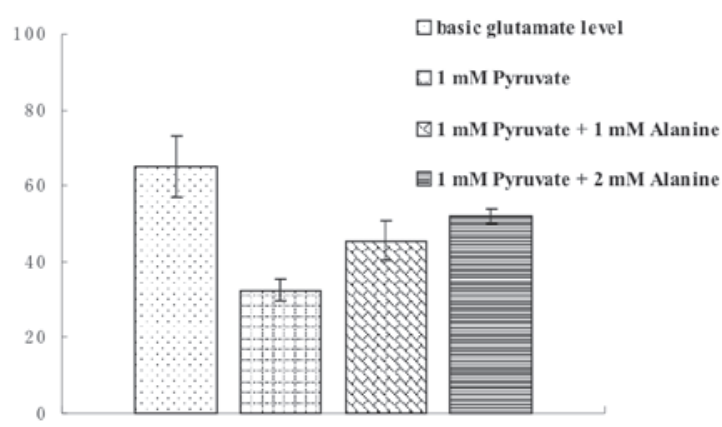

Figure 3. Impact of alanine on glutamate transamination.

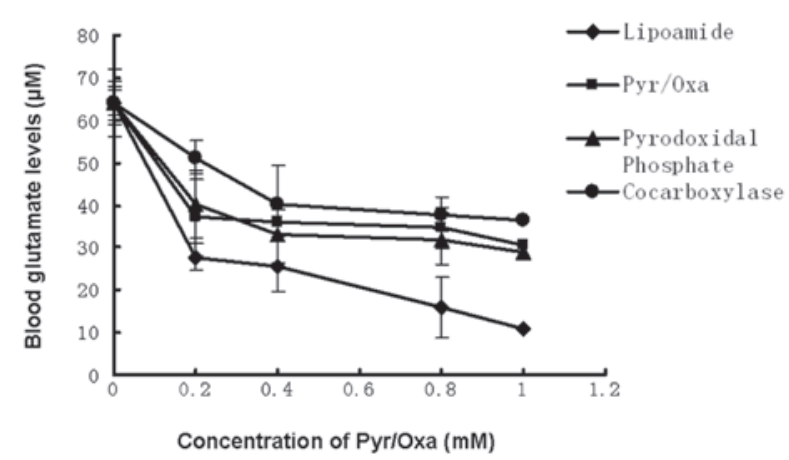

Figure 4. Impact of cofactors together with various concentrations of pyruvate and oxaloacetate (Pyr/Oxa) on glutamate scavenging in blood. Data for one representative experiment out of five performed are shown. 


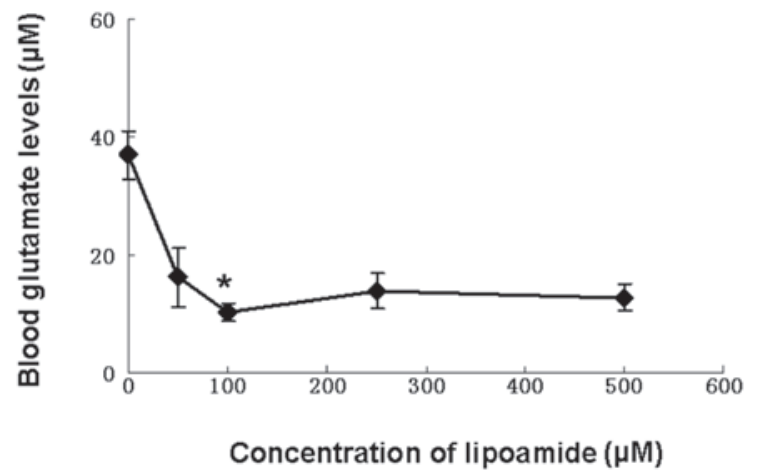

Figure 5. Blood glutamate scavenged by $1 \mathrm{mM}$ pyruvate and oxaloacetate (Pyr/Oxa) together with different concentrations of lipoamide. ${ }^{*} \mathrm{P}<0.05 \mathrm{com}-$ pared with $1 \mathrm{mM}$ Pyr/Oxa alone.

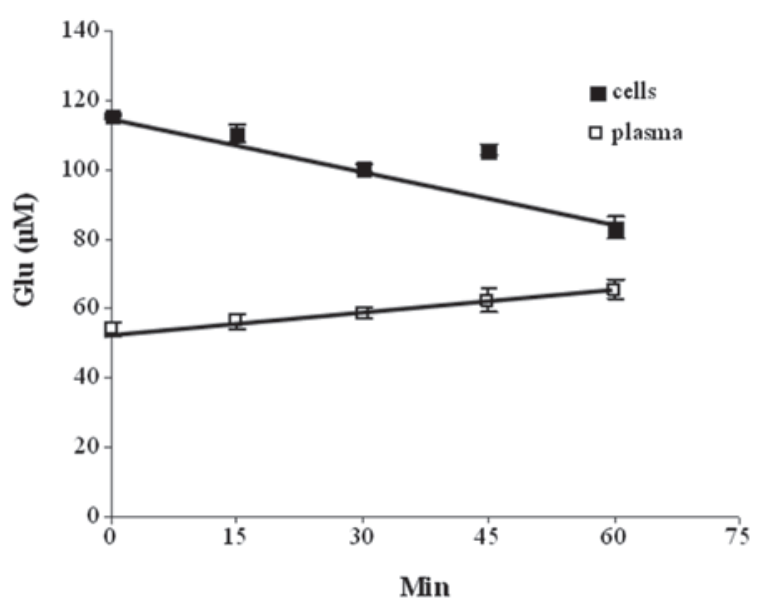

Figure 6. In vitro glutamate evolution in blood plasma and blood cells upon repeated additions of $1 \mathrm{mM}$ nicotinamide adenine dinucleotide (NAD) every $15 \mathrm{~min}$ for $1 \mathrm{~h}$. Glu, glutamic acid.

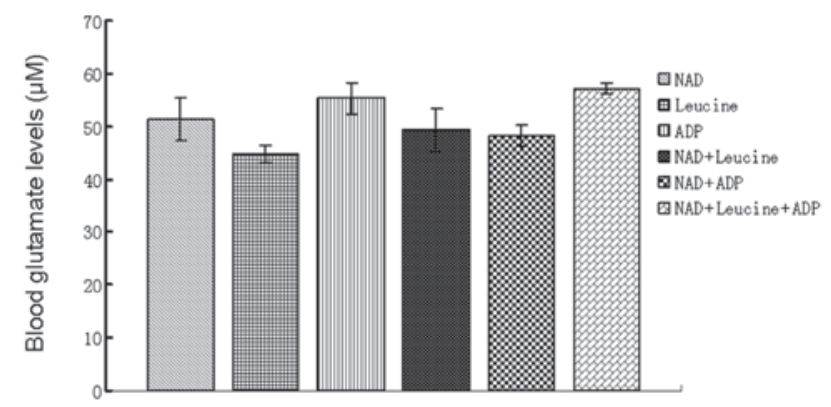

Figure 7. Glutamate levels in blood following repeated addition of $1 \mathrm{mM}$ nicotinamide adenine dinucleotide (NAD), leucine, adenine dinucleotide phosphate (ADP) or their combinations every $15 \mathrm{~min}$ for a total duration of $60 \mathrm{~min}$.

levels occured in the plasma. These results may be interpreted as NAD possibly activating GDH in the cellular compartment, with the $\alpha$-ketoglutarate produced being released from the cells and converted back into glutamate via the GPT or GOT present in the plasma. In all instances of blood being incubated with NAD, leucine or ADP either separately or in combination, increases in plasma glutamate levels occurred (Fig. 7). However, systemic decreases in the cellular compartment were

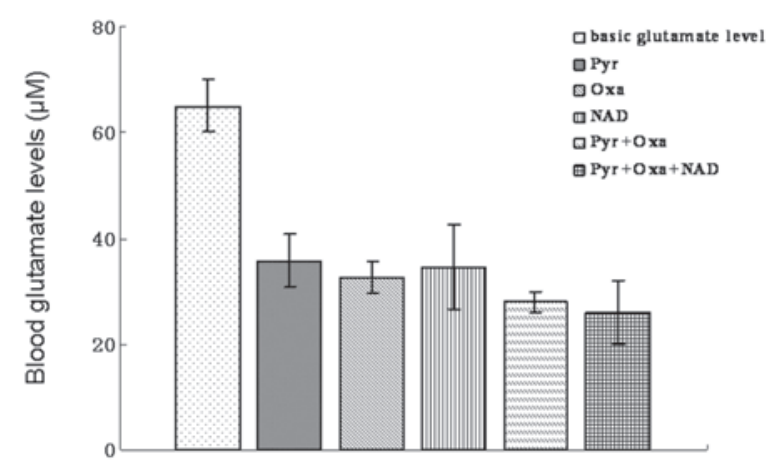

Figure 8. Glutamate levels in blood following repeated addition of $1 \mathrm{mM}$ pyruvate (Pyr), oxaloacetate (Oxa) or nicotinamide adenine dinucleotide (NAD) or their combinations every $15 \mathrm{~min}$ for $60 \mathrm{~min}$.

observed when NAD was present alone or in combination with leucine and ADP, while the latter did not significantly affect glutamate levels alone (data not shown). When blood was incubated with a mixture of NAD, Pyr and Oxa, glutamate levels decreased to a marginally greater extent than with Pyr or Oxa alone (Fig. 8).

Scavenging of blood glutamate by Pyr/Oxa and lipoamide in vivo. Previously, we reported that upon continuous intravenous administration of $\mathrm{Pyr} / \mathrm{Oxa}$, blood, as well as $\mathrm{CSF} /$ interstitial fluid glutamate levels were reduced to $\leq 50 \%$ (4). Since the present in vitro study demonstrated that by administration of lipoamide together with $\mathrm{Pyr} / \mathrm{Oxa}$, it was possible to further reduce blood glutamate levels by $83 \%$ compared with $50 \%$ for $\mathrm{Pyr} / \mathrm{Oxa}$ alone, an in vivo study was performed to examine whether Pyr/Oxa together with lipoamide was able to further reduce blood glutamate levels compared with the effect of Pyr/Oxa alone. Fig. 9 illustrates the effect of Pyr/Oxa alone or together with lipoamide administered continuously through an intravenous catheter at a rate of $50 \mu \mathrm{mol} / \mathrm{min}$ for $\mathrm{Pyr} / \mathrm{Oxa}$ and $100 \mu \mathrm{mol} / \mathrm{min}$ for lipoamide for a duration of 30 min. A marked decrease (55\% for Pyr/Oxa and $70 \%$ for $\mathrm{Pyr} / \mathrm{Oxa} /$ /lipoamide) in blood glutamate occurred after $15 \mathrm{~min}$. This is consistent with the in vitro results. However, as soon as the administration of $\mathrm{Pyr} / \mathrm{Oxa}$ and lipoamide was ceased, the blood glutamate levels increased. When the levels of glutamate were monitored for $\sim 200 \mathrm{~min}$ following the completion of the injection of Pyr/Oxa and lipoamide, glutamate levels clearly exceeded basal blood levels, suggesting that the increase in glutamate levels is not only due to the back reactions as a result of the build-up of the enzymatic products, $\alpha$-ketoglutarate, alanine and aspartate, but possibly to additional compensatory processes causing various organs to release their glutamate content into the plasma to adjust to the novel conditions.

Impact of blood glutamate scavenging on the brain-to-blood glutamate efflux. To monitor the fate of excess glutamate in the CSF, a bolus injection of $10 \mu \mathrm{Ci}\left[{ }^{3} \mathrm{H}\right] \mathrm{Glu}(230$ pmol glutamate $/ 10 \mu \mathrm{l}$ ) was administered to the lateral ventricles of rats and the appearance of radioactivity in the blood was monitored in relation to time. Fig. 10 shows the changes in radioactivity levels in the blood upon intracerebroventricular injection of $10 \mu \mathrm{Ci}\left[{ }^{3} \mathrm{H}\right]$ glutamate followed by blood glutamate scavenging by Pyr/Oxa and lipoamide. As soon as a constant level of 


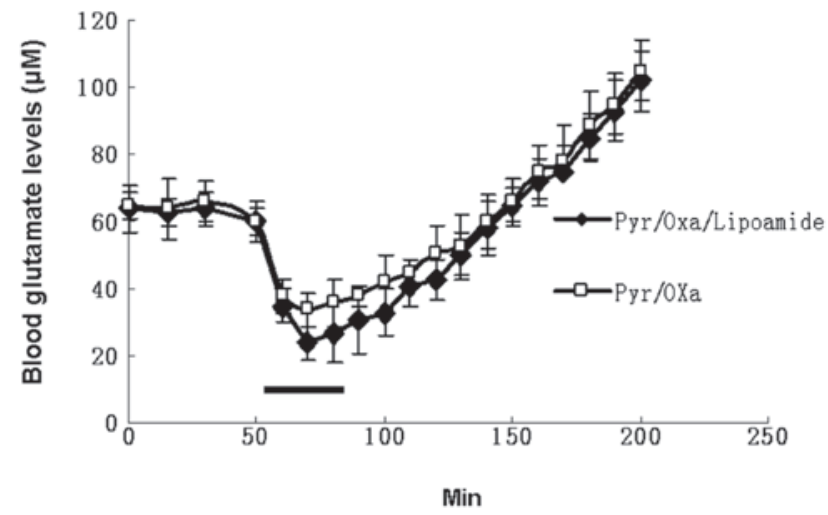

Figure 9. Evolution of blood glutamate levels in vivo upon intravenous administration for a duration of $30 \mathrm{~min}$ (black bar) of pyruvate and oxaloacetate (Pyr/Oxa) alone or with lipoamide.

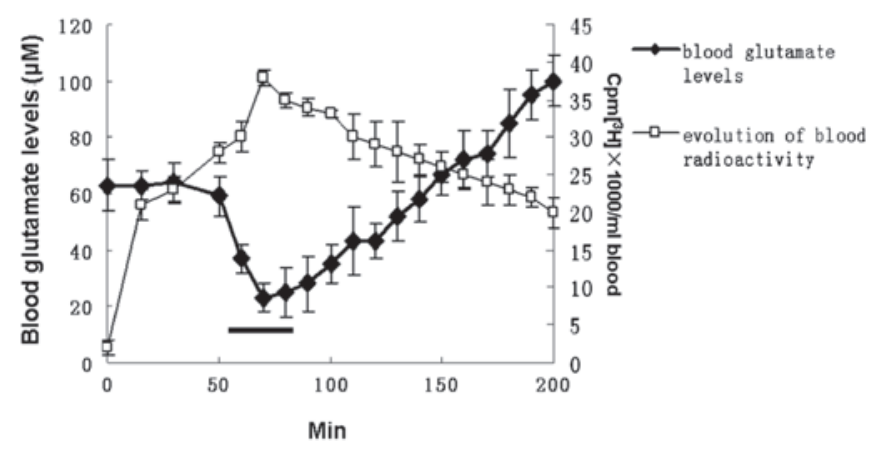

Figure 10. Impact of blood glutamate scavenging by pyruvate/oxaloacetate/lipoamide on the brain-to-blood $\left[{ }^{3} \mathrm{H}\right]$ glutamate efflux upon intravenous administration for a duration of 30 min (black bar).

radioactivity was reached in the blood, blood glutamate levels were transiently decreased by the intravenous administration of Pyr/Oxa and lipoamide. The changes in blood radioactivity levels originating from the brain mirrored those of blood glutamate. While the latter decreased by $\sim 50 \%$ during the administration of Pyr/Oxa with lipoamide and then increased, the blood radioactivity increased by $\sim 40 \%$ and then decreased.

\section{Discussion}

The neuroprotective properties of pyruvate have been thoroughly studied (7-12); however, they have not been used in the clinic. Our previous studies revealed that it is possible to regulate blood glutamate levels by activation of the blood resident enzymes GPT and GOT with their respective substrates Pyr and Oxa (4,5). This blood glutamate decrease was observed to accelerate the efflux of glutamate from the brain to the blood, thereby causing a decrease in glutamate levels in the CSF by increasing the driving force of the brain-to-blood glutamate efflux. Due to the ability of Pyr/Oxa to decrease blood and CSF glutamate levels, these natural GPT and GOT cosubstrates are potentially suitable for therapeutic applications in the context of the large number of neurodegenerative conditions, in which excess CSF glutamate has been indicated to exert a crucial excitotoxicity. In this study, the optimal conditions for Pyr/Oxa to reduce blood glutamate levels were investigated, and numerous cofactors of these reactions for glutamate degeneration were assessed in an attempt to further reduce glutamate levels in blood concomitant with the decrease in glutamate levels in CSF.

The results show that the blood glutamate levels were reduced by $\leq 50 \%$ with $1 \mathrm{mM}$ Pyr/Oxa administered over $60 \mathrm{~min}$. The GTP-catalyzed conversion reaction of glutamate and pyruvate to $\alpha$-ketoglutarate and alanine is reversible. With the accumulation of $\alpha$-ketoglutarate and alanine, the reaction is inhibited. Consistent with this, the results of the present study demonstrate that the addition of alanine inhibits the ability of pyruvate to reduce glutamate levels. $\alpha$-ketoglutarate is the most common product of GPT and GOT. The reduction or removal of $\alpha$-ketoglutarate shifts the reaction towards the conversion of glutamate. $\alpha$-ketoglutarate dehydrogenase is another resident enzyme in the blood, which converts $\alpha$-ketoglutarate into succinyl CoA in the presence of CoA and NAD. It was shown that CoA and NAD have no marked effects on the glutamate conversion by Pyr/Oxa, and cocarboxylase, a cofactor of $\alpha$-ketoglutarate dehydrogenase, decreases glutamate conversion. However, lipoamide, the other cofactor of $\alpha$-ketoglutarate dehydrogenase, exerts a significant effect on blood glutamate levels and decreases them by $>80 \%$ compared with $50 \%$ for $\mathrm{Pyr} / \mathrm{Oxa}$ alone. In the in vivo experiments, blood glutamate levels were reduced to $30 \%$ of the basal level and the efflux of glutamate from the brain to the blood was enhanced upon intravenous administration of Pyr/Oxa and lipoamide.

Lipoic acid is an essential cofactor for numerous enzyme complexes. One of the most visible roles of lipoic acid is as a cofactor in aerobic metabolism, specifically in the pyruvate dehydrogenase complex. Lipoamide is the functional form of lipoic acid in which the carboxyl group is attached to protein by an amide linkage to a lysine amino group. Lipoate was initially termed the pyruvate oxidation factor (POF) by Irwin C. Gunsalus (13). The structure was determined by Gunsalus et al, and the synthetic compound designated as $\alpha$-lipoic acid proved to be the correct molecule (13). Lipoic acid has a number of uses. It has been consumed as a dietary supplement since the early 1990s and has been successfully used for the treatment of chronic liver disease (viral hepatitis, autoimmune hepatitis) (14). A chronic toxicity/carcinogenicity study in rats revealed that racemic lipoic acid was noncarcinogenic and there was no evidence of target organ toxicity (15). In addition, due to the ability of lipoic acid to modify gene expression by stabilizing the transcription factor nuclear factor- $\kappa \mathrm{B}$, Berkson (16) used it for the treatment of various cancers for which no effective treatments existed. Lipoic acid was also suggested to be an effective antioxidant when its ability of preventing the symptoms of vitamin $C$ and vitamin $\mathrm{E}$ deficiency was revealed. Lipoic acid has been shown to have an important neuroprotective role in numerous brain injury conditions (17-25). The present study shows that that lipoamide is able to further decrease blood glutamate levels together with Pyr/Oxa in vivo and in vitro. The results provide further evidence for the neuroprotective role of lipoic acid.

The validity of the neuroprotective strategy of using Pyr/Oxa to reduce blood and CSF glutamate levels has been tested by Dr V.I. Teichberg in several animal models of neurodegenerative conditions, including head trauma, stroke, glioma and exposure to neuronal agents (26). These studies obtained 
promising results, which established the proof of concept that blood glutamate scavenging provides highly effective neuroprotection against acute and chronic neurodegenerative conditions. Lipoamide appears to enhance the effects of $\mathrm{Pyr} / \mathrm{Oxa}$; however, the validity of this finding requires further investigation.

The present study further demonstrated that blood glutamate scavenging enhances glutamate efflux from brain to blood and is therefore likely to provide highly effective neuroprotection against acute and chronic neurodegenerative conditions.

\section{Acknowledgements}

This study was supported by the National Natural Science Foundation of China (grant nos. 31060140, 81060034, 81060126,81060112 and 31260243). The Project Sponsored by the Scientific Research Foundation for the Returned Overseas Chinese Scholars, State Education Ministry and the Program for New Century Excellent Talents in University (NCET), State Education Ministry for Dr Yin Wang

\section{References}

1. Danbolt NC: Glutamate uptake. Prog Neurobiol 65: 1-105, 2001.

2. Basuroy S, Leffler CW and Parfenova H: CORM-A1 prevents blood-brain barrier dysfunction caused by ionotropic glutamate receptor-mediated endothelial oxidative stress and apoptosis. Am J Physiol Cell Physiol 304: C1105-C1115, 2013.

3. Hosoya K, Sugawara M, Asaba H and Terasaki T: Blood-brain barrier produces significant efflux of L-aspartic acid but not $\mathrm{D}$-aspartic acid: in vivo evidence using the brain efflux index method. J Neurochem 73: 1206-1211, 1999.

4. Gottlieb M, Wang Y and Teichberg VI: Blood-mediated scavenging of cerebrospinal fluid glutamate. J Neurochem 87: 119-126, 2003.

5. Wang Y, Gottlieb M and Teichberg VI: An evaluation of erythrocytes as plasma glutamate scavengers for enhanced brain-to-blood glutamate efflux. Neurochem Res 29: 755-760, 2004.

6. Graham LT Jr and Aprison MH: Fluorometric determination of aspartate, glutamate, and gamma-aminobutyrate in nerve tissue using enzymic methods. Anal Biochem 15: 487-497, 1966.

7. Izumi Y, Benz AM, Zorumski CF and Olney JW: Effects of lactate and pyruvate on glucose deprivation in rat hippocampal slices. Neuroreport 5: 617-620, 1994.

8. Matsumoto K, Yamada K, Kohmura E, Kinoshita A and Hayakawa T: Role of pyruvate in ischaemia-like conditions on cultured neurons. Neurol Res 16: 460-464, 1994.

9. Ruiz F, Alvarez G, Pereira R, Hernández M, Villalba M, Cruz F, Cerdán S, Bogónez E and Satrústegui J: Protection by pyruvate and malate against glutamate-mediated neurotoxicity. Neuroreport 9: 1277-1282, 1998.

10. Maus M, Marin P, Israël M, Glowinski J and Prémont J: Pyruvate and lactate protect striatal neurons against N-methyl-D-aspartate-induced neurotoxicity. Eur J Neurosci 11: 3215-3224, 1999.
11. Mongan PD, Fontana JL, Chen R and Bünger R: Intravenous pyruvate prolongs survival during hemorrhagic shock in swine. Am J Physiol 277: H2253-H2263, 1999.

12. Lee JY, Kim YH and KohJ Y: Protection by pyruvate against transient forebrain ischemia in rats. J Neurosci 21: RC171, 2001.

13. Reed LJ, DeBusk BG, Gunsalus IC and Hornberger CS Jr: Crystalline alpha-lipoic acid; a catalytic agent associated with pyruvate dehydrogenase. Science 114: 93-94, 1951.

14. Berkson BM: Thioctic acid in the treatment of poisoning with alpha amanitin. In: Amanita Toxins and Poisonings (International Amanita Symposium, Heidelberg, Nov. 1-3, 1978). Faulstich H, Kommerell B and Wieland T (eds). Verlag Gerhard Witzstrock, Baden-Baden, Koln, New York, p203, 1980.

15. Berkson B: Thioctic acid in treatment of hepatotoxic mushroom (Phalloides) poisoning. N Engl J Med 300: 371, 1979.

16. Cremer DR, Rabeler R, Roberts A and Lynch B: Long-term safety of $\alpha$-lipoic acid (ALA) consumption: A 2-year study. Regul Toxicol Pharm 46: 193-201, 2006.

17. Mainini G, Rotondi M, Di Nola K, Pezzella MT, Iervolino SA, Seguino E, D'Eufemia D, Iannicelli I and Torella M: Oral supplementation with antioxidant agents containing alpha lipoic acid: effects on postmenopausal bone mass. Clin Exp Obstet Gynecol 39: 489-493, 2012.

18. Freitas RM: The evaluation of effects of lipoic acid on the lipid peroxidation, nitrite formation and antioxidant enzymes in the hippocampus of rats after pilocarpine-induced seizures. Neurosci Lett 455: 140-144, 2009.

19. Suchy J, Chan A and Shea TB: Dietary supplementation with a combination of alpha-lipoic acid, acetyl-L-carnitine, glycerophosphocoline, docosahexaenoic acid, and phosphatidylserine reduces oxidative damage to murine brain and improves cognitive performance. Nutr Res 29: 70-74, 2009.

20. Jia Z, Zhu H, Vitto MJ, Misra BR, Li Y and Misra HP: Alpha-lipoic acid potently inhibits peroxynitrite-mediated DNA strand breakage and hydroxyl radical formation: implications for the neuroprotective effects of alpha-lipoic acid. Mol Cell Biochem 323: 131-138, 2009.

21. Karunakaran S, DiwakarL, Saeed U,Agarwal V, RamakrishnanS, Iyengar S and Ravindranath V: Activation of apoptosis signal regulating kinase 1 (ASK1) and translocation of death-associated protein, Daxx, in substantia nigra pars compacta in a mouse model of Parkinson's disease: protection by alpha-lipoic acid. FASEB J 21: 2226-2236, 2007.

22. Zhang L, Xing GQ, Barker JL, Chang Y, Maric D, Ma W, Li BS and Rubinow DR: Alpha-lipoic acid protects rat cortical neurons against cell death induced by amyloid and hydrogen peroxide through the Akt signalling pathway. Neurosci Lett 312: 125-128, 2001.

23. Kim DC, Jun DW, Jang EC, Kim SH, Kim EK, Lee SP, Lee KN, Lee HL, Lee OY, Yoon BC and Choi HS: Lipoic acid prevents the changes of intracellular lipid partitioning by free fatty acid. Gut Liver 7: 221-227, 2013.

24. Connell BJ, Saleh M, Khan BV and Saleh TM: Lipoic acid protects against reperfusion injury in the early stages of cerebral ischemia. Brain Res 1375: 128-136, 2011.

25. Emmez H, Yildirim Z, Kale A, Tönge M, Durdağ E, Börcek AO, Uçankuş LN, Doğulu F, Kiliç N and Baykaner MK: Anti-apoptotic and neuroprotective effects of $\alpha$-lipoic acid on spinal cord ischemia-reperfusion injury in rabbits. Acta Neurochir (Wien) 152: 1591-1601, 2010.

26. Zlotnik A, Gurevich B, Cherniavsky E, Tkachov S, Matuzani-Ruban A, Leon A, Shapira Y and Teichberg VI: contribution of the blood glutamate scavenging activity of pyruvate to its neuroprotective properties in a rat model of closed head injury. Neurochem Res 33: 1044-1050, 2008. 